\title{
EUS for Pancreas Cysts: What Should We Be Sampling?
}

\author{
Jayaprakash Sreenarasimhaiah
}

Published online: 17 March 2013

(c) Springer Science+Business Media New York 2013

The growing resolution and more frequent use of imaging methods has increasingly identified pancreatic cysts, ranging from benign serous and pseudocysts to premalignant or malignant mucinous cystic neoplasm (MCN). Due to the disparate course and management of benign cysts versus cystic neoplasms or pre-neoplasms, unambiguous lesion identification is essential, which is not always possible with conventional cross-sectional imaging [1]. Thus, an increasing number of centers are utilizing endoscopic ultrasound (EUS) for further characterization of pancreatic cysts with high resolution images of cyst morphology, size, presence and type of septations, presence of solid components, and the ability to sample cyst contents using fine needle aspiration (FNA) and to perform biopsies. Characteristics such as diameter $>3 \mathrm{~cm}$ and the presence of mural nodules may be the best predictors of malignant transformation of MCN or side-branch type intraductal papillary neoplasms (SB-IPMN) [2]. In some cases, however, biopsy of solid material or aspiration of cyst fluid is essential to confirm the diagnosis. Thus, when a pancreas cyst is detected on CT or MRI, the clinical question often arises as to whether EUS should be performed and, furthermore, if additional FNA or biopsy is helpful.

In this month's issue of Digestive Diseases and Sciences, Lim et al. [3] report their findings in "Factors determining diagnostic yield of EUS guided fine-needle aspiration for pancreatic cystic lesions: a multicenter Asian study." The authors include a multicenter analysis of 298 patients with diverse pancreatic cystic lesions who underwent EUS of whom 132 (44\%) underwent EUS-FNA.

J. Sreenarasimhaiah $(\bowtie)$

University of Texas Southwestern Medical Center,

Dallas, TX, USA

e-mail: jayaprakash.sree@yahoo.com
FNA cytology with a single pass was only $29 \%$ when solid components are not present. Furthermore, their study demonstrated a higher yield (44\%) when solid components were biopsied during a single pass, which increased to $78 \%$ with multiple passes. Although biochemical markers such as carcinogenic embryonic antigen (CEA) and amylase measured in cyst fluid aspirates are frequently used to aid in diagnosis of pancreatic cysts, the authors focused on cytologic yield from EUS-FNA. In the literature, the overall yield of EUS-FNA for cytologic analysis of indeterminate pancreatic cystic lesions is 31-37\% [2, 4].

Morphologic features noted by high resolution EUS images of small pancreatic cysts can help differentiate mucinous-type cysts from others. Nevertheless, in a recent study, expert endosonographers were evaluated for their ability to make a diagnosis of pancreatic MCN based on EUS appearance alone without FNA. The accuracy was only $23-46 \%$ despite the use septations, mural nodules, solid components, and communication with the pancreatic duct as criteria [5]. Therefore, FNA for fluid analysis or biopsy of solid materials may add to diagnostic yield, particularly for indeterminate cysts. Solid material within a cyst may represent debris but could also arise from malignancy.

Several studies have addressed the acquisition of pancreatic cyst specimens and types of analysis that provide the highest diagnostic yield. Cytologic analysis of aspirated fluid is usually of low yield due to the unlikelihood that pancreatic cysts shed adequate cells for evaluation. Accordingly, a recent study demonstrated that FNA of the cyst wall rather than fluid aspiration alone can improve diagnostic yield up to $81 \%$, with the majority of cases demonstrating mucinous epithelium [6]. Other investigators have examined the use of a cytology brush passed through a 19-gauge FNA needle to gather more cellular 
tissue. Of 30 patients, the brush failed technically in 8 (27\%) but did improve cytologic yield to 76 versus $23 \%$ for fluid aspiration alone, although at the cost of a $10 \%$ complication rate, including one case of pancreatitis, one self-limited bleed, and one case of fatal retroperitoneal bleeding [7]. Thus, this method of sampling is still investigational.

The presence of a solid component in mucinous cysts raises concern for advanced histology with progression to carcinoma [8]. Nonetheless, prior to surgery, biopsy may be helpful to confirm histology and to optimize management. In this study, the authors reported an increase in cytologic yield for solid components in comparison to aspiration of fluid alone. One could argue that biopsies may not be necessary if surgical management is the next appropriate step. Yet, some patients (and clinicians) will require concrete evidence of malignancy before invoking surgical therapeutic options.

Traditional teaching has been to perform a single pass into a cystic lesion with attempt at complete evacuation of the cyst contents, which can often be difficult in multiloculated cysts filled with viscous material. On the other hand, the authors demonstrated the safety of multiple passes into different compartments of a cyst in addition to multiple biopsies of solid components. More importantly, they demonstrated an increase in cytologic yield. Although larger needles such as 19 or 22 gauge may be needed to aspirate viscous fluid contents, complete evacuation of all cyst contents in every multi-loculated pancreatic cyst with solid components remains challenging, with increased risk of infection and hemorrhage. Thus, this technique may need further evaluation to determine its feasibility and safety.

The utility of biomarkers such as CEA, amylase, CA 19-9 and DNA mutational analysis of the cyst contents has been reported in several studies but remains preliminary. Since the amount of fluid that is obtained from cyst aspiration is usually quite limited, it is often necessary to choose between biomarker assay or cytologic analysis. Further studies addressing the test characteristics of biomarkers and advances in micro-analysis should make biomarker analysis an increasingly routine and valuable adjunct to cytology.

Is EUS truly needed for the evaluation of pancreatic cysts? Furthermore, when and how should biopsies be performed? As cysts are discovered more and more frequently as incidental findings on routine cross-sectional images, the clinical scenario should be carefully considered. Since highly morbid surgery is the only effective intervention for most pancreatic cystic neoplasms, the patient's surgical candidacy should be considered before embarking on invasive investigations. EUS has become well established as one of the best modalities with very high resolution images to determine accurate size, morphology, and concerning features such as mural nodules. The most important aspect of managing an indeterminate pancreatic cyst is to establish if it is of a mucinous type and to characterize its malignant potential. Small asymptomatic cysts may require no further intervention. Conversely, solid components and especially mural nodules can raise concerns of malignancy. Such pancreatic cysts may benefit from EUS-guided biopsy but should be correlated to the clinical presentation. As Lim et al. [3] suggest in this study, the cytologic yield of fluid alone remains poor, but FNA is useful especially with multiple passes when solid components are present. Accordingly, further studies are needed to test the hypothesis that EUS-FNA may not be needed in the absence of solid components, although all other cysts may still benefit from a diagnostic EUS imaging study.

Finally, advances in cytologic techniques and analysis such as immunohistochemistry and flow cytometry are likely to improve diagnostic yield. We look to more powerful tests of pancreatic cyst aspirates that include cytology and multiple biochemical markers on low volume specimens. Until then, endosonographers should sample wisely to get the most out of small lesions.

\section{References}

1. Adimoolam V, Sanchez MJ, Siddiqui UD, et al. Endoscopic ultrasound identifies synchronous pancreas cystic lesions not seen on initial cross-sectional imaging. Pancreas. 2011;40:1070-1072.

2. Hawes RH, Clancy J, Hasan MK. Endoscopic ultrasound-guided fine needle aspiration in cystic pancreatic lesions. Clin Endosc. 2012;45:128-131.

3. Lim LG, Lakhtakia S, Ang TL, et al. Factors determining diagnostic yield of endoscopic ultrasound guided fine-needle aspiration for pancreatic cystic lesions: a multicenter Asian study. Dig Dis Sci. (Epub ahead of print). doi:10.1007/s10620-0122528-2.

4. de Jong K, Poley JW, van Hooft JE, Visser M, Bruno MJ, Fockens $P$. Endoscopic ultrasound-guided fine-needle aspiration of pancreatic cystic lesions provides inadequate material for cytology and laboratory analysis: initial results from a prospective study. Endoscopy. 2011;43:585-590.

5. de Jong K, Verlaan T, Dijkgraaf MG, et al. Interobserver agreement for endosonography in the diagnosis of pancreatic cysts. Endoscopy. 2011;43:579-584.

6. Hong SK, Loren DE, Rogart JN, et al. Targeted cyst wall puncture and aspiration during EUS-FNA increases the diagnostic yield of premalignant and malignant pancreatic cysts. Gastrointest Endosc. 2012;75:775-782.

7. Sendino O, Fernández-Esparrach G, Solé M, et al. Endoscopic ultrasonography-guided brushing increases cellular diagnosis of pancreatic cysts: a prospective study. Dig Liver Dis. 2010;42:877.

8. Ohno E, Hirooka Y, Itoh A, et al. Intraductal papillary mucinous neoplasms of the pancreas: differentiation of malignant and benign tumors by endoscopic ultrasonography findings of mural nodules. Ann Surg. 2011 (Epub ahead of print). doi: 10.1097/SLA.0b013 e31819edle5. 